\title{
Curva de crescimento em altura na cernelha de equinos da raça Mangalarga Marchador considerando-se heterocedasticidade
}

\author{
[Growth curve for height at withers of Mangalarga Marchador horses with heterocedasticity] \\ R.A. Ribeiro ${ }^{1}$, F.A.C. Souza ${ }^{2}$, J.A. Muniz ${ }^{3}$, T.J. Fernandes ${ }^{3}$, R.S. Moura ${ }^{3}$ \\ ${ }^{1}$ Aluna de graduação - Universidade Federal de Lavras - Ufla - Lavras, MG \\ ${ }^{2}$ Aluno de pós-graduação - Universidade Federal de Lavras - Ufla - Lavras, MG \\ ${ }^{3}$ Universidade Federal de Lavras - Ufla - Lavras, MG
}

\begin{abstract}
RESUMO
Objetivou-se, com este estudo, avaliar o ajuste dos modelos de Brody, Gompertz, Logístico e Von Bertalanffy aos dados de altura na cernelha de equinos Mangalarga Marchador, ponderando pelo inverso da variância, a fim de selecionar o melhor modelo e predizer sobre o crescimento e a maturidade dos animais dessa raça. Foram utilizados dados de 230 equinos dos seis aos 176 meses de idade, os quais foram divididos por sexo e em 16 classes de idade. Os modelos estudados foram comparados segundo os avaliadores de qualidade: coeficiente de determinação $\left(\mathrm{R}^{2}\right)$; desvio padrão residual (DPR) e critério de informação de Akaike (AIC). A estimação dos parâmetros dos modelos foi realizada pelo método de mínimos quadrados ponderado no software R. Todos os modelos avaliados se mostraram adequados para descrever a curva de crescimento em ambos os sexos. Perante os avaliadores utilizados, o modelo Logístico foi o mais adequado para descrever as curvas de crescimento em altura na cernelha nos dois sexos da raça Mangalarga Marchador. Observou-se também um crescimento acelerado nos primeiros meses de idade. Os animais machos atingiram uma altura adulta maior, porém as fêmeas são mais precoces, pois apresentaram maior estimativa para o índice de maturidade.
\end{abstract}

Palavras-chave: idade, logístico, medidas morfométricas, modelos não lineares

\begin{abstract}
This work aimed to evaluate the fit of models Brody, Gompertz, Logistic and Von Bertalanffy to height at the withers of Mangalarga Marchador horses, weighed by the inverse of variance, in order to select the best model and predict growth and maturity of this breed pf animals. We used data of 230 equines from 06 to 176 months of age who were divided by sex and 16 age classes. The models were compared according to the quality assessors: coefficient of determination $\left(R^{2}\right)$; residual standard deviation (RSD) and Akaike information criteria (AIC). The estimation of the parameters from models was performed by the weighted least squares method in Software $R$. All models evaluated were suitable to describe the growth curve in both sexes. In view of the evaluators used, the logistic model was the most suitable to describe the growth curves in withers height in both sexes for the race Mangalarga Marchador. A strong growth at first age was also observed. The males reached a greater adult height, but females did so sooner, because they presented higher estimates for the maturity index.
\end{abstract}

Keywords: age, logistic, morphometric measurements, nonlinear models

\section{INTRODUÇÃO}

A raça Mangalarga Marchador (MM) originou-se na região sul do estado de Minas Gerais, sendo considerada a maior raça equina nacional. Possui como principais características grande beleza

Recebido em 28 de setembro de 2016

Aceito em 10 de fevereiro de 2017

E-mail: rribeiro17@outlook.com zootécnica, docilidade, rusticidade e aptidão para o lazer, os esportes e o trabalho agropecuário. A versatilidade dos equinos MM, aliada a sua conformação e andamento marchado, são características que levam os animais a atingirem elevados preços no mercado (Costa et al., 2004; Santiago et al., 2016). 
Conhecer o padrão de desenvolvimento da raça permite a estimativa de parâmetros que possibilitam selecionar, com acurácia elevada, os animais ainda em idade precoce, a fim de otimizar o manejo de criatórios. Muitos problemas relacionados ao crescimento dos equinos podem ser prevenidos quando se dispõe do conhecimento da curva de crescimento da raça e de suas variações, uma vez que alguns cavalos podem apresentar taxa de desenvolvimento (crescimento ósseo e muscular) mais lento do que outros, mesmo estando coordenado o seu sistema de crescimento (Santos et al., 2007).

A altura na cernelha, entre todas as medidas lineares, é uma das mais seguras, por mostrar menores variações decorrentes do instrumento de mensuração ou dos erros cometidos pelo mensurador (Cabral et al., 2004). Um dos primeiros levantamentos do registro de altura na cernelha da raça Mangalarga Marchador foi feito por Carneiro et al. (1952), em que os valores médios de altura na cernelha para os machos foram de $151 \mathrm{~cm}$ e para as fêmeas, de $144 \mathrm{~cm}$. Atualmente, para efeito de registro definitivo dos equinos na Associação Brasileira de Criadores do Cavalo Mangalarga Marchador (Estatuto da ABCCMM, 2000), os valores aceitos são de 147 a $157 \mathrm{~cm}$ nos machos, e nas fêmeas de 140 a $154 \mathrm{~cm}$.

Nos estudos envolvendo o crescimento e desenvolvimento de diversas espécies animais, os modelos não lineares têm se mostrado adequados por apresentarem bons ajustes e fornecerem estimativas de parâmetros com interpretação biológica (Carneiro et al., 2014; Mota et al., 2015; Veloso et al., 2016). Quando o conjunto de dados em estudo apresenta heterogeneidade de variâncias, a estimação dos parâmetros de modelos não lineares ponderandose pelo inverso da variância é recomendada (Pasternak e Shalev, 1994). Essa ponderação faz com que as medidas com maior variância tenham menor peso no processo de estimação, o que reduz o erro padrão das estimativas dos parâmetros, levando a resultados mais confiáveis (Prado et al., 2013; Carvalho et al., 2014; Fernandes et al., 2014; Muianga et al., 2016).

Poucos estudos são direcionados para o crescimento e desenvolvimento equino, devido às dificuldades de obtenção dos dados, uma vez que o crescimento dessa espécie se apresenta mais tardio em relação a outras. Alguns trabalhos, como Santos et al. (1999) e Santos et al. (2007), foram conduzidos para obter a descrição do crescimento de cavalos pantaneiros. McManus et al. (2010) avaliaram o crescimento de animais de quatro grupos genéticos criados pelo Exército brasileiro. Fraldinho et al. (2015) estudaram o desenvolvimento da raça Puro Sangue Lusitano, a fim de entender a precocidade de machos e fêmeas e proporcionar melhorias nas condições de manejo dos animais. No entanto, um aspecto importante a ser destacado é a falta de trabalhos que descrevem o crescimento de medidas morfométricas, como a altura na cernelha, principalmente na raça Mangalarga Marchador.

Este estudo objetivou avaliar o ajuste dos modelos Brody, Gompertz, Logístico e Von Bertalanffy, ponderados pelo inverso da variância, aos dados de altura na cernelha de equinos Mangalarga Marchador, a fim de selecionar o melhor modelo e predizer $\mathrm{o}$ crescimento e a maturidade de machos e fêmeas dessa raça.

\section{MATERIAL E MÉTODOS}

Os dados analisados foram obtidos em 2012, durante um evento no Parque de Exposições Bolívar de Andrade, em Belo Horizonte/MG. O estudo foi aprovado pelo Comitê de Ética em Pesquisa com Animais, sob protocolo 039/12. A altura na cernelha, medida do ponto mais alto da região interescapular até o solo, foi mensurada em 96 machos e 134 fêmeas da raça Mangalarga Marchador, os quais foram divididos em 16 classes de idade e por sexo (macho e fêmea).

Foram utilizados os modelos de regressão não lineares de Brody (1), Gompertz (2), Logístico (3) e von Bertalanffy (4) para descrever a curva de crescimento em altura na cernelha dos equinos:

$$
\begin{array}{r}
\mathrm{y}_{\mathrm{i}}=\mathrm{a} *\left\{1-\exp \left[\mathrm{k}\left(\mathrm{t}_{\mathrm{i}}-\mathrm{b}\right)\right]\right\}+\varepsilon_{\mathrm{i}} \\
\mathrm{y}_{\mathrm{i}}=\mathrm{a} * \exp \left\{-\exp \left[\mathrm{k}\left(\mathrm{t}_{\mathrm{i}}-\mathrm{b}\right)\right]\right\}+\varepsilon_{\mathrm{i}} \\
\mathrm{y}_{\mathrm{i}}=\frac{\mathrm{a}}{\left\{1-\exp \left[\mathrm{k}\left(\mathrm{t}_{\mathrm{i}}-\mathrm{b}\right)\right]\right\}(1)}+\varepsilon_{\mathrm{i}}
\end{array}
$$




$$
\mathrm{y}_{\mathrm{i}}=\mathrm{a} *\left\{1-\exp \left[1\left(\mathrm{t}_{\mathrm{i}}-\mathrm{b}\right)\right]\right\}^{3}+\varepsilon_{\mathrm{i}}
$$

Nas equações (1), (2), (3) e (4), tem-se que: " $y_{i}$ " é a altura na cernelha, em $\mathrm{cm}$; " $a$ " é o valor assintótico, ou altura na cernelha na idade adulta do animal; " $b$ " está associado ao ponto de inflexão do modelo, ou seja, idade em que o animal passa de um crescimento acelerado para um crescimento desacelerado até estabilizar em seu valor máximo $a$; " $k$ " está associado ao crescimento da altura na cernelha, é o índice de precocidade, uma vez que quanto maior o valor de $k$, menos tempo será necessário para o animal atingir a sua altura adulta; " $t$ " refere-se à idade em que o animal foi mensurado; e " $\varepsilon_{i}$ " é o erro experimental associado ao modelo, que se pressupõe ser aleatório, independente e com distribuição normal $\mathrm{N}\left(0, \sigma^{2}\right)$.

Por meio do teste de Durbin-Watson, analisou-se a possível presença de dependência residual entre as medidas, sendo avaliado se o resíduo de uma observação está associado com o resíduo das observações adjacentes. Verificou-se também a pressuposição de normalidade pelo teste de Shapiro-Wilk e o pressuposto de homocedasticidade residual por meio do teste de Breusch-Pagan.

Foi calculada a variância entre os animais dentro de uma mesma classe de idade, possibilitando, assim incorporar essa variabilidade no processo de estimação dos parâmetros, por meio da ponderação pelo inverso da variância, como sugerido por Pasternak e Shalev (1994). A estimação dos parâmetros dos modelos foi realizada, portanto, pelo método de mínimos quadrados ponderado, utilizando-se o algoritmo numérico de Gauss-Newton. Sob a suposição de normalidade dos resíduos, foram obtidos também os intervalos de confiança de $95 \%$ para as estimativas dos parâmetros.

Os modelos foram comparados quanto à qualidade do ajuste fornecido, o que possibilitou indicar qual o mais adequado para descrever a curva de crescimento em função da idade dos equinos da raça Mangalarga Marchador para a variável altura na cernelha. Os critérios de qualidade de ajuste utilizados foram:

i.desvio-padrão residual (DPR), calculado pela expressão, $\mathrm{DPR}=\sqrt{\mathrm{QME}}$ sendo $\mathrm{QME} \quad \mathrm{o}$ quadrado médio residual. Quanto menor for o DPR melhor o modelo;

ii. coeficiente de determinação $\left(R^{2}\right)$, obtido por: , $\mathrm{R}^{2}=1-\frac{\mathrm{SQE}}{\text { SQtotal }}$ em que SQE é a soma de quadrados dos resíduos e SQTotal é a soma de quadrados total. O coeficiente de determinação varia de 0 a 1 , tendo melhor ajuste o modelo cujo $\mathrm{R}^{2}$ estiver mais próximo de 1 ;

iii. critério de informação de Akaike (AIC) \{ TC "3.5.4 Critério de Informação de Akaike (AIC)" If C $\backslash 1$ "3" \}, calculado por meio da expressão:

$\mathrm{AIC}=-2$ Loglike $+2 \mathrm{p}$, em que o Loglike corresponde ao logaritmo de base natural da função da verossimilhança e $p$ a quantidade de parâmetros do modelo. O modelo escolhido será aquele que apresentar menor valor de $A I C$.

As estimativas para os parâmetros dos modelos, bem como os ajustes gráficos e toda a parte computacional envolvida na elaboração deste trabalho, foram obtidas utilizando-se o software estatístico R (R Development Core team, 2015).

\section{RESULTADOS E DISCUSSÃO}

Os animais foram divididos em classes de acordo com a sua faixa etária e por sexo, conforme Tab. 1 , de modo que se obteve a média e a variância de cada classe. $O$ uso da ponderação na estimação dos parâmetros foi necessário, pois percebeu-se uma grande heterogeneidade entre as variâncias amostrais nas classes de idade. 
Curva de crescimento em altura...

Tabela 1. Médias e variâncias calculadas em cada classe de idade e em ambos os sexos

\begin{tabular}{ccccccc}
\hline \multirow{2}{*}{$\begin{array}{c}\text { IDADE } \\
\text { (meses) }\end{array}$} & \multicolumn{2}{c}{$\mathrm{N}^{\circ}$ de animais } & \multicolumn{2}{c}{ Machos } & \multicolumn{2}{c}{ Fêmeas } \\
\cline { 2 - 6 } $6-8$ & 4 & 7 & Média & Variância & Média & Variância \\
$9-11$ & 12 & 11 & 125,5143 & 62,1653 & 123,4286 & 19,5355 \\
$12-14$ & 2 & 3 & 127,2512 & 15,1243 & 132,3333 & 7,5828 \\
$15-17$ & 2 & 2 & 138,7689 & 10,2981 & 133,5276 & 12,4997 \\
$18-20$ & 8 & 8 & 140,5625 & 8,5310 & 139,3753 & 12,7677 \\
$21-23$ & 7 & 10 & 140,6429 & 25,3089 & 141,1035 & 21,9332 \\
$24-29$ & 3 & 2 & 139,6667 & 42,5821 & 146,5012 & 5,2654 \\
$30-35$ & 6 & 13 & 146,4167 & 2,7413 & 144,3929 & 17,9682 \\
$36-41$ & 4 & 2 & 145,3751 & 29,2291 & 147,5765 & 40,4992 \\
$42-47$ & 8 & 10 & 146,5625 & 14,4597 & 147,6123 & 11,0995 \\
$48-53$ & 2 & 4 & 148,5102 & 4,1235 & 145,8751 & 7,7289 \\
$54-59$ & 9 & 16 & 150,6502 & 4,0024 & 148,6875 & 5,1624 \\
$60-71$ & 7 & 13 & 150,0129 & 16,7493 & 145,6538 & 18,8069 \\
$72-83$ & 9 & 8 & 147,2222 & 5,6316 & 145,7515 & 28,0709 \\
$84-95$ & 3 & 8 & 148,5017 & 10,7498 & 150,2523 & 17,2142 \\
$96-176$ & 10 & 17 & 149,2579 & 24,5688 & 146,7941 & 22,6890 \\
\hline
\end{tabular}

Os resultados do teste de Durbin-Watson não foram significativos e, portanto, aceita-se a hipótese de independência entre os resíduos. Tal fato pode ser explicado por terem sido observados animais provenientes de diferentes criatórios, caracterizando um estudo transversal de crescimento. O teste de Shapiro-Wilk constatou a normalidade dos resíduos para altura na cernelha, e o teste de Breusch-Pagan indicou homocedasticidade residual. $\mathrm{O}$ grande tamanho amostral estudado pode ter sido o responsável por essa aceitação de todos os pressupostos em relação aos resíduos.

As estimativas para o parâmetro $a$, o qual mede a altura adulta estimada, apresentaram-se um pouco maiores nos machos (cerca de $149 \mathrm{~cm}$ ) que nas fêmeas (cerca de $147 \mathrm{~cm}$ ), com uma diferença média de $2 \mathrm{~cm}$, como pode ser visualizado nas Tab. 2 e 3. Gonçalves et al. (2012) afirmaram que tal variação pode ser relacionada com o dimorfismo sexual existente entre os sexos, visto que os machos possuem características sexuais secundárias diferentes das fêmeas, o que influencia o crescimento de regiões corporais e, assim, favorece uma estatura maior.

O padrão racial da raça Mangalarga Marchador aprovado pela ABCCMM preconiza como altura na cernelha ideal para as fêmeas $146 \mathrm{~cm}$, admitindo-se para o registro definitivo altura mínima de $140 \mathrm{~cm}$ e máxima de $154 \mathrm{~cm}$. Já para os machos, a altura ideal é $152 \mathrm{~cm}$, admitindo-se para registro definitivo a mínina de $147 \mathrm{~cm}$ e a máxima de $157 \mathrm{~cm}$. As estimativas encontradas pelo presente estudo condizem com o padrão ideal, uma vez que estão contidas no intervalo proposto pela ABCCMM. Tal ocorrência pode ser explicada pelo fato de os dados terem sido obtidos em uma exposição oficial da raça, na qual todos os animais presentes tinham registro, o que justifica a interpretação prática dos parâmetros estimados.

Tabela 2. Estimativas dos parâmetros e limites inferior (LI) e superior (LS) dos intervalos de confiança para os parâmetros dos quatro modelos na descrição da curva de crescimento em altura na cernelha de fêmeas da raça Mangalarga Marchador

\begin{tabular}{ccccrrrrrr}
\hline \multirow{2}{*}{ Modelos } & \multicolumn{10}{c}{ Parâmetros } \\
\cline { 2 - 10 } & LI & $a$ & LS & LI & $k$ & LS & LI & $b$ & LS \\
\hline Brody & 146,4412 & 147,9234 & 149,4112 & 0,0631 & 0,0881 & 0,1121 & $-19,1991$ & $-12,9742$ & $-6,7491$ \\
Gompertz & 146,4323 & 147,8756 & 149,3010 & 0,0682 & 0,0934 & 0,1184 & $-16,5052$ & $-10,9113$ & $-5,3172$ \\
Logístico & 146,4256 & 147,8267 & 149,2135 & 0,0723 & 0,0988 & 0,1243 & $-14,0813$ & $-9,0283$ & $-3,9746$ \\
Bertalanffy & 146,4428 & 147,8992 & 149,3412 & 0,0665 & 0,0916 & 0,1162 & $-32,5384$ & $-9,0280$ & $-3,9748$ \\
\hline
\end{tabular}

Legenda: $a=$ valor assintótico, ou altura na cernelha adulta do animal; $b=$ parâmetro associado ao ponto de inflexão do modelo; $k=$ índice de maturidade ou de precocidade do modelo; $\mathrm{LI}=$ limite inferior do intervalo de confiança; $\mathrm{LS}=$ limite superior do intervalo de confiança. 
Tabela 3. Estimativas dos parâmetros e limites inferior (LI) e superior (LS) dos intervalos de confiança para os parâmetros dos quatro modelos na descrição da curva de crescimento em altura na cernelha de machos da raça Mangalarga Marchador

\begin{tabular}{cccccccccc}
\multirow{2}{*}{ Modelos } & \multicolumn{10}{c}{ Parâmetros } \\
\cline { 2 - 10 } & LI & $a$ & LS & LI & $k$ & LS & LI & $b$ & LS \\
\hline Brody & 147,5223 & 149,3923 & 151,2523 & 0,0471 & 0,0736 & 0,0991 & $-25,8121$ & $-16,3971$ & $-6,9821$ \\
Gompertz & 147,5245 & 149,3245 & 151,1256 & 0,0515 & 0,0779 & 0,1045 & $-22,4042$ & $-13,9544$ & $-5,5042$ \\
Logístico & 147,5134 & 149,2656 & 151,0127 & 0,0546 & 0,0823 & 0,1094 & $-19,3606$ & $-11,7276$ & $-4,0955$ \\
Bertalanffy & 147,5278 & 149,3478 & 151,1639 & 0,0509 & 0,0764 & 0,1023 & $-42,7428$ & $-29,1099$ & $-15,4779$ \\
\hline
\end{tabular}

Legenda: $a=$ valor assintótico, ou altura na cernelha adulta do animal; $b=$ parâmetro associado ao ponto de inflexão do modelo; $k=$ índice de maturidade ou de precocidade do modelo; $\mathrm{LI}=$ limite inferior do intervalo de confiança; $\mathrm{LS}=$ limite superior do intervalo de confiança.

O valor estimado para altura na cernelha adulta para fêmeas (parâmetro $a$, Tab. 2) de aproximadamente $147 \mathrm{~cm}$ está próximo do ideal proposto pelo padrão racial de $146 \mathrm{~cm}$. Já os machos apresentaram estimativa em torno de $149 \mathrm{~cm}$ (parâmetro $a$, Tab. 3), a qual se encontra dentro do intervalo aceitável, porém mais próxima do limite inferior de $147 \mathrm{~cm}$. Tal resultado corrobora ao de Santiago et al. (2016), que, ao estudarem a evolução das medidas morfométricas da raça Mangalarga Marchador entre 1970 e 2010, também perceberam que, no decorrer dos anos, a altura na cernelha das fêmeas foi se aproximando do ideal de $146 \mathrm{~cm}$; já os machos tendem a subestimar o valor de $152 \mathrm{~cm}$ ao longo das décadas.

Ainda se comparando os resultados encontrados por Santiago et al. (2016) com os do presente estudo, observa-se que a altura na cernelha na idade adulta para fêmeas (Tab. 2) continua com uma tendência de crescimento, ao passo que, para os machos, as estimativas para a altura na cernelha permanecem constantes, semelhantes aos valores observados por esses autores.

Menezes et al. (2014), ao estudarem a altura na cernelha na raça Quarto de Milha, também encontraram valores maiores nos machos, porém suas medidas são menores que as descritas neste trabalho com a raça Mangalarga Marchador. O valor encontrado pelos autores foi de $147,7 \mathrm{~cm}$ nos machos, o que sugere que a raça Quarto de milha pode apresentar altura na cernelha menor quando comparada à Mangalarga Marchador.

Ao se analisarem os valores encontrados para os intervalos de confiança de $95 \%$, percebe-se que os limites inferiores e superiores, em ambos os sexos, estão contidos dentro dos limites preconizados pela $\mathrm{ABCCMM}$, o que indica uma grande confiança nas estimativas dos parâmetros, uma vez que todos os animais estudados estão dentro das normas definidas pelo padrão racial da ABCCMM.

As fêmeas tendem a atingir a sua altura na cernelha adulta em um espaço de tempo menor que os machos, ou seja, mais precocemente, pois as estimativas do parâmetro $k$ foram maiores nas fêmeas que nos machos nos respectivos modelos, como visualizado na Tab. 2. Esse resultado corrobora ao de Santos et al. (1999), que avaliaram a curva de crescimento para a medida altura na cernelha de cavalos Pantaneiros e observaram que os machos apresentaram um valor adulto superior ao das fêmeas, porém o valor do índice de maturidade $(k)$ estimado também foi inferior nos machos.

Como pode ser observado na Tab. 4, os modelos de regressão não linear forneceram ótimos ajustes na descrição da curva de crescimento, pois apresentaram $\mathrm{R}^{2}$ superior aos $93 \%$, sendo os melhores modelos, segundo os avaliadores de qualidade, o Gompertz e o Logístico em ambos os sexos. Porém, entre eles, o que apresentou maior valor de $\mathrm{R}^{2}$ e menores valores de $\mathrm{AIC}$ e DPR foi o Logístico. Assim, esse modelo é o mais indicado para representar a curva de crescimento da variável altura na cernelha de equinos da raça Mangalarga Marchador em ambos os sexos. O modelo Logístico também é o mais indicado para estudar curvas de crescimento em outras espécies, como nos trabalhos de Silva et al. (2012), Mota et al. (2015) e Veloso et al. (2016) na descrição da curva de crescimento de cabras, codornas e frangos de corte, respectivamente. 
Tabela 4. Valores do coeficiente de determinação $\left(R^{2}\right)$, desvio-padrão residual (DPR) e critério de informação de Akaike (AIC), na avaliação do ajuste dos modelos de crescimento da altura na cernelha de machos e fêmeas da raça Mangalarga Marchador

\begin{tabular}{ccccc}
\hline Modelos & Sexo & $\mathrm{R}^{2}$ & DPR & AIC \\
\hline Brody & Machos & 0,9375 & 1,1734 & 71,4628 \\
& Fêmeas & 0,9510 & 0,9522 & 70,1745 \\
Gompertz & Machos & 0,9387 & 1,1599 & 71,1161 \\
& Fêmeas & 0,9523 & 0,9393 & 69,7393 \\
Logístico & Machos & 0,9396 & 1,1481 & 70,8104 \\
& Fêmeas & 0,9535 & 0,9275 & 69,3335 \\
Von Bertalanffy & Machos & 0,9383 & 1,1642 & 71,2271 \\
& Fêmeas & 0,9519 & 0,9435 & 69,8811 \\
\hline
\end{tabular}

Ao avaliarem a altura na cernelha para cavalos Pantaneiros, Santos et al. (2007) concluíram que o melhor modelo foi o de Richards. McManus et al. (2010) avaliaram animais das raças Hanoveriana (HA), Brasileiro de Hipismo (BH), Puro Sangue Inglês (PSI) e mestiços (PSIxBH) criados pelo Exército brasileiro e encontraram como melhor modelo o de Brody na avaliação da altura na cernelha. Já Fraldinho et al. (2015) indicaram o modelo Richards para descrever a curva de crescimento de equinos da raça Lusitano. Percebe-se, portanto, que existe uma diferença no padrão de crescimento de equinos de raças distintas, pois os modelos de crescimento indicados diferiram em cada uma delas, o que ressalta a importância de se estudar o crescimento de cada raça separadamente e, por conseguinte, justifica a relevância deste trabalho.
Como pode ser observado na Fig.1, os animais apresentaram um crescimento acelerado nos primeiros 24 meses de idade, e, principalmente nessa fase, o modelo Logístico apresenta uma ótima qualidade de ajuste. A altura dos potros ao nascimento representa cerca de $60 \%$ da altura adulta, e o crescimento dessa medida morfométrica é muito intenso até um ano de idade, sendo uma das principais preocupações dos criadores acompanhar esse crescimento para tomar decisões adequadas de seleção e manejo (Santos et al., 2007). O acelerado crescimento nos primeiros meses de idade também foi observado por Cabral et al. (2004), que encontraram valores correspondentes ao ganho médio de altura na cernelha de $20 \mathrm{~cm}$ no primeiro trimestre de vida do animal; já no segundo, terceiro e quarto trimestres, foi de $9 \mathrm{~cm}, 6 \mathrm{~cm} \mathrm{e}$ $4,6 \mathrm{~cm}$, respectivamente.
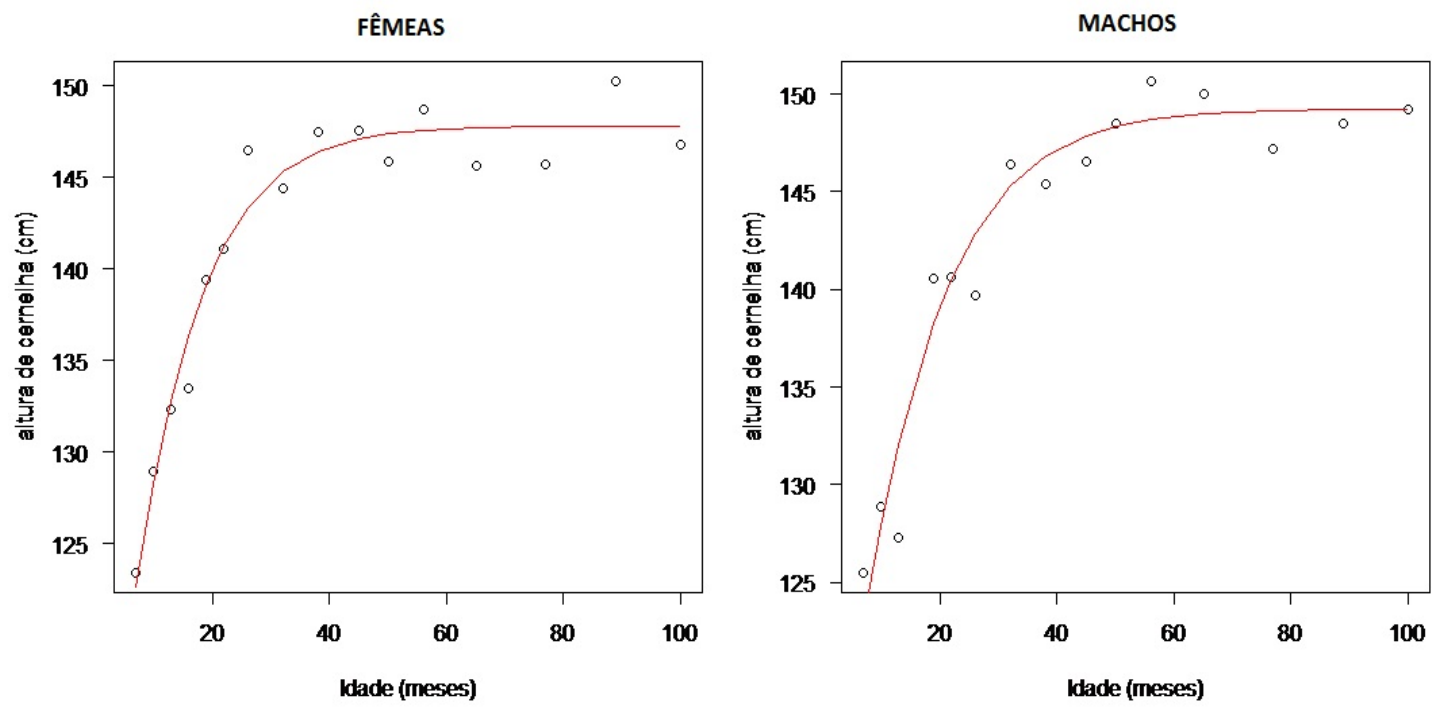

Figura 1. Representação da curva de crescimento da altura na cernelha obtida pelo modelo não linear Logístico para fêmeas e machos da raça Mangalarga Marchador. 


\section{CONCLUSÃO}

O modelo de regressão não linear Logístico, ponderado pelo inverso da variância, é o mais indicado para descrever o crescimento em altura na cernelha de animais Mangalarga Marchador de ambos os sexos. Observou-se um crescimento muito acelerado nos primeiros 24 meses de idade. Os machos adultos apresentam altura na cernelha maior que as fêmeas, no entanto elas são mais precoces, pois apresentaram maior estimativa para o índice de maturidade.

\section{REFERÊNCIAS}

CABRAL, G.C.; ALMEIDA, F.Q.D.; QUIRINO, C.R. et al. Avaliação morfométrica de equinos da raça Mangalarga Marchador: conformação e proporções corporais. Rev. Bras. Zootec., v.33, p.1798-1805, 2004.

CARNEIRO, A.P.S.; MUNIZ, J.A.; CARNEIRO, P.L.S. et al. Identidade de modelos não lineares para comparar curvas de crescimento de bovinos da raça Tabapuã. Pesqui. Agropecu. Bras., v.49, p.57-62, 2014.

CARNEIRO, G.G.; FONTES, L.R.; PEREIRA, H.C. Informações preliminares sobre o cavalo Mangalarga Marchador. Arch. Esc. Sup. Vet., v.5, p.5-9, 1952.

CARVALHO, L.R.; PEREIRA, G.M.S.; SILVA, H.D.O.F. et al. Ajustes de modelos não lineares de efeitos fixos, com ponderação e misto-aplicações. Rev. Bras. Biometric, v.32, p.296-307, 2014.

COSTA, M.D.; BERGMANN, J.A.G.; REZENDE, A.S.C. et al. Demographic characterization of Mangalarga Marchador breed. Arq. Bras. Med. Vet. Zootec., v.56, p.687-690, 2004.

ESTATUTO: padrão da raça, Belo Horizonte: ABCCMM, 2000. 8p. Disponível em: $<$ http://www.abccmm.org.br/regulumantos $>$. Acessado em: 15 jun. 2016.

FERNANDES, T.J.; PEREIRA, A.A.; MUNIZ, J.A.; SAVIAN, T.V. Seleção de modelos não lineares para a descrição das curvas de crescimento do fruto do cafeeiro. Coffee Sci., v.9, p.207-215, 2014

FRALDINHO, M.J.; BESSA, R.J.B.; FERREIRADIAS, G.; CALDEIRA, R.M. Growth and development of the Lusitano horse managed on grazing systems. Livest. Sci., v.186, p.22-28, 2015.

GONÇALVES, R.W.; COSTA, M.D.; REZENDE, A.S.C. et al. Efeito da endogamia sobre características morfométricas em cavalos da raça Mangalarga Marchador. Arq. Bras. Med. Vet. Zootec., v.64, p.419426, 2012.
MCMANUS C.M., HELDER LOUVANDINI H.; CAMPOS V.A.L. Non linear growth curves for weight and height in four genetic groups of horses. Cienc. Anim. Bras., v.11, p.80-89, 2010.

MENESES, A.C.A.; COSTA, M.D.; MARUCH, S. et al. Medidas lineares e angulares de animais da raça Quarto de Milha utilizados em uma prova de vaquejada. Rev. Bras. Cienc. Vet., v.21, p.256-261, 2014

MOTA, L.F.M.; ALCÂNTARA, D.C.; ABREU, L.R.A. et al. Crescimento de codornas de diferentes grupos genéticos por meio de modelos não lineares. Arq. Bras. Med. Vet. Zootec., v.67, p.1372-1380, 2015.

MUIANGA, C.A.; MUNIZ, J.A.; NASCIMENTO, M.D.S. et al. Description of the growth curve of cashew fruits in nonlinear models. Rev. Bras. Fruticul. v.38, p.22-32, 2016

PASTERNAK, H.; SHALEV, B.A. The effect of a feature of regression disturbance on the efficiency offitting growth curves. Growth Dev. Agin, v.58, p.3339, 1994.

PRADO, T.K.L.; MUNIZ, J.A.; SAVIAN, T.V.; SÁFADI, T. et al. Ajuste do modelo logístico na descrição do crescimento de frutos de coqueiro anão por meio de algoritmos iterativos MCMC. Rev. Bras. Biometria, v.31, p.216-232, 2013.

$\mathrm{R}$ DEVELOPMENT core team: a language and environment for statistical computing. Vienna: R Foundation for Statistical Computing, 2015. Available in: <http://www.r-project.org $>$. Accessed in: 21 Aug. 2015.

SANTIAGO, J.M.; REZENDE, A.S.C.D.; LANA, A.M.Q. et al. Evolution of morphometric measures in the Mangalarga Marchador breed. Rev. Caatinga, v.29, p.191-199, 2016.

SANTOS, S.A.; OLIVEIRA, M.R.D.; SERENO, J.R. Using nonlinear models to describe heigth growth curves in pantaneiro horses. Pesqui. Agropecu. Bras. v.34, p.1133-1138, 1999

SANTOS, S.A.; SOUZA, G.S.; ABREU, U.G.P. et al. Monitoramento do desenvolvimento de cavalos pantaneiros por meio de curvas de crescimento. Arch. Zootec., v.56, Sup.1, p.648, 2007.

SILVA, L.S.A.; FRAGA, A.B.; SILVA, F.L. et al. Growth curve in Santa Inês sheep. Small Ruminant Res., v.105, p.182-185, 2012.

VELOSO, R.C.; WINKELSTROTER, L.K.; SILVA, M.T.P. et al. Seleção e classificação multivariada de modelos não lineares para frangos de corte. Arq. Bras. Med. Vet. Zootec., v.68, p.191-200, 2016. 